\title{
The HST view of the nuclear emission line region in low luminosity radio-galaxies ${ }^{\star}$
}

\author{
A. Capetti ${ }^{1}$, G. V. Kleijn ${ }^{2}$, and M. Chiaberge ${ }^{3}$ \\ 1 INAF - Osservatorio Astronomico di Torino, Strada Osservatorio 20, 10025 Pino Torinese, Italy \\ e-mail: capetti@to.astro.it \\ 2 European Southern Observatory, Karl-Schwarzschild-Strasse 2, 85748 Garching, Germany \\ e-mail: gverdoes@eso.org \\ 3 INAF - Istituto di Radioastronomia, via Gobetti 101, 40129 Bologna, Italy \\ e-mail: chiab@ira.cnr.it
}

Received 7 July 2004 / Accepted 5 April 2005

\begin{abstract}
We study the properties of the emission line regions in two samples of low luminosity radio-galaxies, while focusing on the Compact Emission Line Region (CELR) revealed to be a characteristic feature of these objects by HST narrow-band imaging. We find a strong correlation between line and optical continuum nuclear emission, which suggests that the optical cores (most likely of non-thermal origin) can be directly associated to the source of ionizing photons, i.e. that we are seeing a jet-ionized narrow line region. A photon budget argument indicates that the optical nuclear sources produce sufficient photon flux provided that the covering factor of the circum-nuclear gas is rather large, on average $\sim 0.3$. Analysis of HST images and spectra suggests that the CELR may take the form of a pc-scale, high filling factor structure, possibly an optically thin torus. Estimates of the CELR mass lead to values as small as $10-10^{3} M_{\odot}$, and photon counting sets a limit to the Broad Line Region mass of $M_{\mathrm{BLR}}<10^{-2} M_{\odot}$. When considered together with the low accretion rate and the tenuous torus structure, a general paucity of gas in the innermost regions of low luminosity radio-galaxies emerges as the main characterizing difference from more powerful Active Galactic Nuclei.
\end{abstract}

Key words. galaxies: active - galaxies: nucleus - galaxies: jets

\section{Introduction}

The study of low luminosity active galactic nuclei (LLAGN) (i.e., bolometric luminosity typically $\lesssim 10^{42} \mathrm{erg} \mathrm{s}^{-1}$ ) is a key tool in improving our understanding of the process of accretion onto super-massive black holes. The LLAGN provide us with a view of the physical mechanisms related to mass accretion in a regime that is complementary to (and possibly different from) those at work in high luminosity AGN (HLAGN). The LLAGN represent a link between the high luminosity AGN and the population of quiescent galaxies, as it is now widely recognized that most galaxies host a super-massive black-hole. Exploring the different manifestations of nuclear activity across the largest possible range of luminosity is then a crucial step towards better understanding the connections between active and non-active galaxies.

LLAGN are found in bright galaxies at a much higher rate than HLAGN. In a sample of nearby galaxies studied by Ho et al. (1997) 30\% show a measurable level of

^ Based on observations obtained at the Space Telescope Science Institute, which is operated by the Association of Universities for Research in Astronomy, Incorporated, under NASA contract NAS 5-26555. nuclear activity, based on the presence of emission lines of nonstellar origin. Recently, this high fraction has been confirmed by analysis of SDSS spectra of a much larger sample (Miller et al. 2003). Radio-loud AGN, which are the focus of this paper, are associated almost invariably with bright elliptical galaxies; their radio-luminosity function indicates that again $\sim 30 \%$ of the galaxies with $M_{\mathrm{V}}<-21$ are associated to radio-sources brighter than $10^{29.5} \mathrm{erg} \mathrm{s}^{-1} \mathrm{~Hz}^{-1}$ (Auriemma et al. 1977).

LLAGN, both radio-quiet and radio-loud, then represent the bulk of active galaxies (and a substantial fraction of the overall galaxies' population). Unfortunately, their study has been significantly hampered by contamination from host galaxy emission which, in most observing bands, dominates emission from the AGN. To constrain the physical processes at work in these objects it is clearly necessary to disentangle the AGN and host's contributions via spectral decomposition or high resolution imaging. In the last few years, thanks to the HST and, more recently, to Chandra imaging, this has become routinely possible down to the lowest end of the AGN luminosity function, at least in the nearby Universe.

An example of the importance of this approach comes from recent results obtained for low luminosity radio-galaxies 
(LLRG). HST broad-band imaging of the sample formed by the 3CR radio-galaxies with FR I morphology revealed, in the majority of the targets, the presence of unresolved optical nuclei (Chiaberge et al. 1999, hereafter CCC99). Fluxes and luminosities of these sources show tight correlation with the radio cores, extending over four orders of magnitude. This has been interpreted as due to a common non-thermal emission process in the radio and optical band, i.e. that we are seeing the optical emission from the base of a relativistic jet. This interpretation is supported by evidence of anisotropy in the optical sources by their spectral indices and, when available, by their spectral energy distributions (Chiaberge et al. 2001, 2003). The dominance of the non-thermal emission and corresponding limits to emission from the accretion disk, might imply a profoundly different nuclear structure with respect to classical, more luminous AGN. The high fraction (85\%) of objects with detected optical nuclear sources suggests a general lack of obscuring molecular tori, a further distinction with respect to other classes of AGN.

Verdoes Kleijn et al. (2002a, hereafter VK02) reached similar conclusions from the observations of another sample of LLRG selected from the UGC catalogue (see Sect. 2 for the sample definition). They also noticed the characteristic presence of a compact nuclear component of emission line, in all but one case (namely, UGC 6635 characterized by a complex dusty morphology). Extended emission is also often detected and almost always associated with dust. Nonetheless the CELR represents in most cases the dominant component, being a fraction of at least $10 \%$ of the line flux within $1 \mathrm{kpc}$, with a median fraction of $70 \%$. They found a close correlation between the radio core and nuclear line emission luminosities.

These observations focusing on the properties of the emission line regions set the stage for a better understanding the physics of low luminosity radio-galaxies. Here we build on the results obtained by Verdoes Kleijn et al. when combining the UGC FR I sample with the FR I galaxies from the 3CR sample for which we analyze HST narrow-band images and spectra, thus extending the coverage in radio-luminosity. Similar to the UGC sample, the 3CR FR I sources show the ubiquitous presence of CELRs. But the higher detection rate of optical nuclei in the $3 \mathrm{C}$ with respect to the UGC sample enables us to perform a more detailed analysis of the relationship between the different emission components in LLRG. The aim of this paper is to establish the source of ionization of CELRs in LLRG and to constrain the physical properties of the CELRs.

We decided here to focus our study on the central component of line emission, although additional extended line emission is often present, as it represents a well-defined structure recognizable in all examined LLRG and thus characteristic feature associated to the nuclear activity of these objects. By limiting ourselves to the nuclear line component, we aim at minimizing the contamination by line-emitting mechanisms not directly related to the active nucleus, such as the extended emission line disk of M 87, which is most likely powered by internal shocks (Dopita et al. 1997) or by clumps of line emission associated to star formation (e.g. O'Dea et al. 2001).

The paper is organized as follows: in Sect. 2 we describe the samples selected for our analysis and the available HST observations. In Sect. 3 we study the relationships between the line luminosities with other relevant properties of our objects which are then used to explore i) the ionization mechanism of the CELR (Sect. 4), ii) its physical structure (Sect. 5) and iii) the possible presence (and origin) of the classical correlation between line and extended radio luminosity in LLRG (Sect. 6). Our results are discussed in Sect. 7 and summarized in Sect. 8.

Throughout this paper, a Hubble constant of $H_{0}=$ $75 \mathrm{~km} \mathrm{~s}^{-1} \mathrm{Mpc}^{-1}$ is adopted.

\section{The samples and HST observations}

For our analysis we selected two samples of low luminosity radio-galaxies. The first is formed by the 33 radio-galaxies with FR I morphology, part of the $3 \mathrm{C}$ catalogue ${ }^{1}$. The second is the sample of 21 radio-bright UGC galaxies from Noel-Storr et al. (2003) defined as E/SO galaxies brighter than both $m_{B}<14.6$ in the optical and $150 \mathrm{mJy}$ at $1400 \mathrm{MHz}$ (see their paper for the precise sample definition). The $3 \mathrm{C}$ sample is selected at low radio frequency and thus is essentially free from orientation biases. Concerning the UGC sample, the selection at $1.4 \mathrm{GHz}$ does not warrant an unbiased selection. However, the radio cores from VLA $1490 \mathrm{MHz}$ measurements (FWHM 1'.5-3".75) always constitute less than $22 \%$ of the total $1400 \mathrm{MHz}$ flux and typically $\sim 6 \%$ (Xu et al. 2000), indicating that orientation can only have a marginal effect on the sample selection.

As there are 7 objects in common, the total number of objects in the combined sample is 47 . The $3 \mathrm{C}$ sample alone spans the range $10^{30.5}<\log L_{\text {ext }}\left[\mathrm{W} \mathrm{Hz}^{-1}\right]<10^{33.5}$ in radio luminosity, but its lowest end is only scarcely populated. Only by including of the UGC sample (all UGC sources have $\log L_{\text {ext }}<$ $10^{32.2}$ ) is the coverage at all levels of radio-luminosity sufficiently uniform to adequately explore the different behaviour over the whole luminosity range spanned by LLRG.

For the whole UGC sample both narrow-band and medium resolution HST spectra are available in addition to broadband imaging. Emission-line measurements of the Compact Emission Line Region (CELR) have been obtained from narrow-band imaging by Verdoes Kleijn et al. (1999), isolating the contribution of the central line component, as well as from the spectra (Noel-Storr et al. 2003). Since the linewidths in most objects are such that de-blending of $\mathrm{H} \alpha$ from the [N II] lines cannot be performed accurately from the medium resolution spectra and since the narrow-band filters do not allow separation of these lines, we always measured the fluxes from the whole $\mathrm{H} \alpha+[\mathrm{N}$ II] complex. Measurements from images and spectra show generally a very close agreement, within less than a factor of 2 , except for a few objects for which the spectroscopic target acquisition failed; we therefore decided to consider, when available, those obtained from spectra.

For nine 3C/FRI sources not part of the VK02 sample, we retrieved all STIS spectra and narrow-band images available from the Multimission Archive at the Space Telescope Science Institute (see Table 1 for a summary).

\footnotetext{
1 Here we include NGC 6251 in the 3C sample, following its revision by Laing et al. (1983); conversely 3C 84 with its ill-defined and peculiar radio-morphology has been removed from the original list.
} 
Table 1. Datasets used for this work.

\begin{tabular}{lccccc}
\hline \hline Name & Program ID & Data type & Instrument & Filter/Grating & Exp. Time \\
\hline 3C 029 & GO8700 & spectrum & STIS-CCD & $G 750 L$ & $360 \mathrm{~s}$ \\
3C 78 & GO8700 & spectrum & STIS-CCD & $G 750 L$ & $720 \mathrm{~s}$ \\
3C 83.1 & GO5957 & image & WFPC2 & $F 673 N$ & $600 \mathrm{~s}$ \\
3C 277.3 & GO8700 & spectrum & STIS-CCD & $G 750 L$ & $360 \mathrm{~s}$ \\
& GO5957 & image & WFPC2 & $F R 680 N$ & $600 \mathrm{~s}$ \\
3C 338 & GO8700 & spectrum & STIS-CCD & $G 750 L$ & $360 \mathrm{~s}$ \\
& GO5957 & image & WFPC2 & $F 673 N$ & $600 \mathrm{~s}$ \\
3C 346 & GO8700 & spectrum & STIS-CCD & $G 750 L$ & $360 \mathrm{~s}$ \\
3C 442 & GO5927 & image & WFPC2 & $F 673 N$ & $2500 \mathrm{~s}$ \\
3C 465 & GO8700 & spectrum & STIS-CCD & $G 750 L$ & $360 \mathrm{~s}$ \\
& GO5957 & image & WFPC2 & $F 673 N$ & $600 \mathrm{~s}$ \\
NGC 6251 & GO6653 & image & WFPC2 & $F 673 N$ & $5700 \mathrm{~s}$ \\
\hline
\end{tabular}

All spectra were taken as part of program GO 8700 with the G750L grating (centered at $7751 \AA$, it covers the spectral range 5240-11490 $\AA$ and it has an average dispersion of $4.92 \AA$ per pixel) in combination with a $0.2^{\prime \prime}$ width slit, centered on the unresolved nucleus of each galaxy. The observations were performed in snapshot mode with a total exposure time of $360 \mathrm{~s}$ (720 s in the case of 3C 78), split into two exposures to allow cosmic-ray rejection. The spectra were calibrated by the standard On The Fly Reprocessing (OTFR) system, but we further processed the $2 \mathrm{D}$ spectra with the IRAF task cosmicrays to eliminate residual cosmic rays and hot pixels that were not removed by the OTFR.

Narrow-band images are available for 6 objects. Four targets were observed as part of the $3 \mathrm{C}$ emission-line snapshot program (GO 5957) using the $F 673 N$ narrow filter (FR680N ramp filter for 3C 277.3) with an exposure time of 600 split into two exposures. The two remaining objects, 3C 442 and NGC 6251, were also observed with the $F 673 \mathrm{~N}$ filter but for different programs with exposure times of 2500 and $5700 \mathrm{~s}$, respectively. All images were calibrated by the OTFR. Continuum removal was obtained by subtracting a scaled version of the $F 702 \mathrm{~W}$ broad-band image ( $F 555 \mathrm{~W}$ for NGC 6251), appropriately scaled by taking into account exposure time and filter sensitivity. The narrow-band, continuum subtracted ${ }^{2}$ images (or the 2D spectra when no images are available) are shown in Fig. 1 revealing the presence of a clearly defined CELR in all 9 objects.

We performed the measurements of the CELR fluxes following a strategy as similar as possible to the one used for the UGC sources. For measurements from narrow-band imaging we extracted the $\mathrm{H} \alpha+[\mathrm{NII}]$ flux of the line component within an aperture of radius 0 .' 1 ; from the spectra, we extracted one-dimensional nuclear spectra by summing over three pixel rows, corresponding to 0.3 , centered on the peak of the emission through the 0.2 slit. We then measured the flux of the

\footnotetext{
${ }^{2}$ For 3C 83.1 the broad-band image is affected by the diffraction spikes of a bright nearby star. Therefore, we cannot determine the continuum subtraction outside the nucleus.
}

$\mathrm{H} \alpha+[\mathrm{NII}]$ emission line complex using the IRAF task splot. For the 3 objects with both spectra and images, the resulting $\mathrm{H} \alpha+[\mathrm{NII}]$ agrees within better than a factor of 2. Spectral measurements are generally more accurate as they have uncertainties ranging from $30 \%$ in the case of $3 \mathrm{C} 28$ to about $15 \%$ for NGC 6251 while those derived from the images have uncertainties between 25 and $50 \%$, so overall similar to those of the VK02 sample.

Before we proceed, there are a few points worth discussing. The selection for the observations of 16 3CR sources for which we have line data are biased toward a significantly lower redshift than the complete FR I/3CR sample, being limited almost exclusively to sources with $z<0.1$, with only 1 exception. It must be noted that none of the (few) $3 \mathrm{C}$ objects without optical core detection has been observed in line emission, so we cannot quantitatively explore possible differences from nucleated galaxies. Conversely, several UGC sources show a CELR where no optical core is seen: most likely, isolating the small spectral range of the $\mathrm{H} \alpha+[\mathrm{N} \mathrm{II}]$ complex enables us to increase the contrast against the host galaxy with respect to broad-band imaging and to isolate the nuclear emission at even lower levels of activity. As no optical, radio, or emission-line core fluxes are detected in UGC 6635, no optical core was detected in UGC 8419 and in UGC 11718, and no measurement for the radio core of UGC 7115 is available; likewise the number of representative points for the UGC sources can vary in the different diagrams.

Results from broad-band HST imaging are presented by CCC99 and VK02 for the 3C and UGC samples respectively. Note that for some 3C sources CCC99 the continuum broad-band fluxes were not corrected for the line contribution as this information was not available. The measurements of the CELR fluxes (corresponding to equivalent widths between 200 and $600 \AA$ ) confirm the typical fractional contribution between 10 and $30 \%$ of the broad-band fluxes, a contamination negligible for our purposes.

We take the values of the optical nuclear fluxes from the above papers as well as the radio measurements they collected 

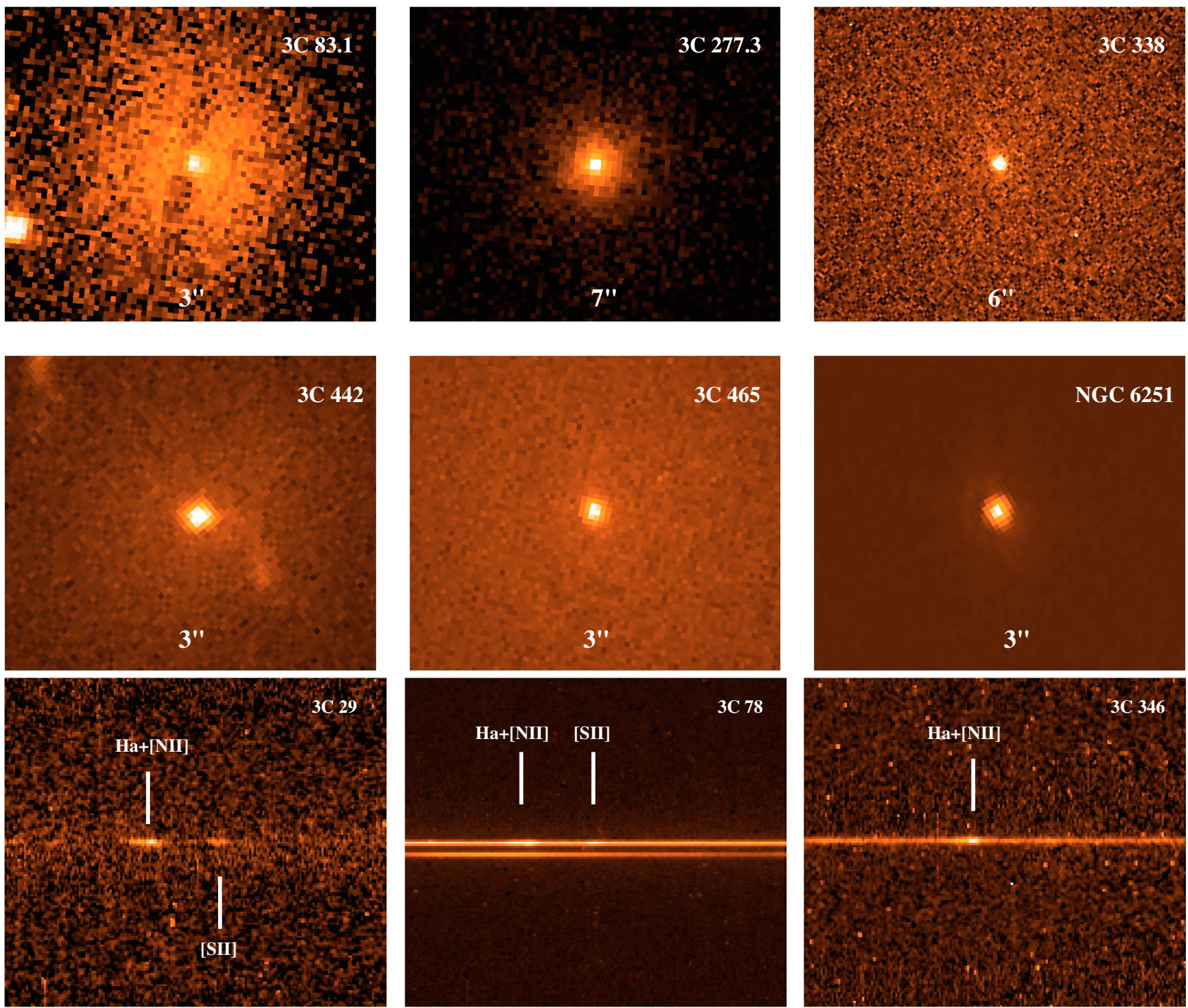

Fig. 1. Continuum subtracted narrow-band images (upper six panels, North points up) or 2D long slit spectra (lower three panels) for nine $3 \mathrm{C}$ sources not in common with the VK02 sample. In all cases the images are dominated by the presence of a compact emission line region. The three spectra show that line emission is spatially unresolved. For 3C 83.1 we present the narrow-band image, without continuum subtraction, see the text.

from the literature. Total radio luminosity is taken at the lowest energy available (between 178 and $1400 \mathrm{MHz}$ ), while radio cores come in most cases from $5 \mathrm{GHz}$ VLBI data. For consistency we converted optical core luminosities to $7020 \AA$ (adopting when necessary a spectral index ${ }^{3}$ of 1 ) and the extended radio luminosities to $178 \mathrm{MHz}$ (adopting a spectral index of 0.7 ).

\section{The relationships between line emission and the radio/optical properties}

In this section we explore the relationships between the line luminosities and other nuclear properties, such as the optical nuclear luminosity, the core and extended radio emission.
In Fig. 2 we compare the $\mathrm{H} \alpha+[\mathrm{N}$ II] and nuclear optical luminosities. A clear correlation is found with a correlation coefficient of 0.77 . The slope of the correlation, derived as the bisectrix of the linear fits using the two quantities as independent variables, is $m=0.85 \pm 0.13$ and the rms of the residuals is 0.45 dex. Slope and intercept are derived from the 3C sources alone (as the bisectrix method cannot be used in presence of upper limits) but UGC sources are clearly consistent with the same correlation. A similar correlation $(r=0.71$, $m=0.56 \pm 0.18$ ) is also found between line and continuum nuclear fluxes, which suggests that the correlation found when considering luminosities is not driven by the common dependence on distance alone. To set this point on a more quantitative ground we estimated the partial correlation coefficient between line and optical nuclear emission excluding the dependence on

\footnotetext{
${ }^{3}$ We define the spectral index $\alpha$ with the spectrum in the form $F_{v} \propto v^{-\alpha}$.
} 
Table 2. Correlations summary.

\begin{tabular}{llcccc}
\hline \hline Var. A & Var. B & $r_{\mathrm{AB}}$ & $r_{\mathrm{AB}, z}$ & Slope & rms \\
\hline$L_{\mathrm{H} \alpha}+[\mathrm{N} \mathrm{II}]$ & $L_{\mathrm{opt}}$ & 0.77 & 0.75 & $0.85 \pm 0.13$ & 0.45 \\
$F_{\mathrm{H} \alpha}+[\mathrm{N} \mathrm{II}]$ & $F_{\mathrm{opt}}$ & 0.71 & - & $0.56 \pm 0.18$ & 0.41 \\
$L_{\mathrm{H} \alpha}+[\mathrm{N} \mathrm{II}]$ & $L_{\text {core }}$ & 0.86 & 0.79 & $0.79 \pm 0.10$ & 0.39 \\
$F_{\mathrm{H} \alpha}+[\mathrm{N} \mathrm{II}]$ & $F_{\text {core }}$ & 0.81 & - & $0.63 \pm 0.13$ & 0.36 \\
$L_{\mathrm{H} \alpha}+[\mathrm{N} \mathrm{III}]$ & $L_{\mathrm{ext}}$ & 0.69 & 0.29 & $1.07 \pm 0.14$ & 0.46 \\
$F_{\mathrm{H} \alpha}+[\mathrm{N} \mathrm{II}]$ & $F_{\mathrm{ext}}$ & 0.40 & - & $0.98 \pm 0.18$ & 0.41 \\
\hline
\end{tabular}

redshift $^{4}: r$ is only marginally reduced to 0.75 which gives a probability $P=8 \times 10^{-4}$ that these 16 points are taken from a random distribution.

The large range of redshift spanned by our objects (from 0.0037 to 0.162 ) raises the issue that the physical size of the extraction region used for the measurements of the line emission varies substantially from object to object. To test the relevance of this problem we repeated the analysis restricting it to a sub-sample of 23 objects with $0.01<z<0.03$; although this reduces the range of line luminosities from 3.5 to $2.5 \mathrm{dex}$, the correlation between $L_{\mathrm{H} \alpha}+[\mathrm{N} \mathrm{II}]$ and $L_{0}$ is still present with no significant changes of the fit parameters.

Using ground based measurements of line intensity collected by Zirbel \& Baum (1995) the results are also essentially unchanged. Restricting to the 9 sources common to both the Zirbel \& Baum and HST samples the correlation has a slope of $0.82 \pm 0.15$ with the normalization a factor of $\sim 2.3$ larger.

A similarly tight correlation is found by comparing the radio core and emission line luminosity, as already found by VK02 for the UGC sample, with a correlation coefficient of 0.86 and a slope of $0.79 \pm 0.10$ (see Fig. 3). This result is somewhat expected on the basis of the strong correlation between optical and radio emission in the cores of FR I radiogalaxies. This comparison is nonetheless interesting mainly due to the higher completeness of detection of radio cores with respect to the optical ones particularly for the UGC sample. This shows that the correlation also holds at the lowest levels of nuclear luminosity, smoothly extending from the $3 \mathrm{C}$ to the UGC sample. The scatter of the correlation between core and line luminosity is slightly smaller $(0.39$ vs. 0.45$)$ than for the optical core. Again, the correlation between radio core and $\mathrm{H} \alpha+[\mathrm{N}$ II $]$ is also present when considering fluxes instead of luminosities, while the correlation coefficient removing the effects of redshift is 0.79 ( $P=4 \times 10^{-7}$ for 29 points).

A correlation between $\mathrm{H} \alpha+[\mathrm{N} \mathrm{II}]$ and extended radio luminosity is also present (see Fig. 4), but in this case the partial correlation coefficient is substantially smaller, 0.29 , indicating that it is mostly driven by their common dependence on redshift.

${ }^{4}$ Given three variables, if the correlation coefficient between $x_{i}$ and $x_{j}$ is $r_{i j}$, the partial correlation coefficient between two of them, excluding the effect of the third variable is given by (see e.g. Kendall $\&$ Stuart 1979)

$r_{12,3}=\frac{r_{12}-r_{13} r_{23}}{\sqrt{1-r_{13}^{2}} \sqrt{1-r_{23}^{2}}}$.
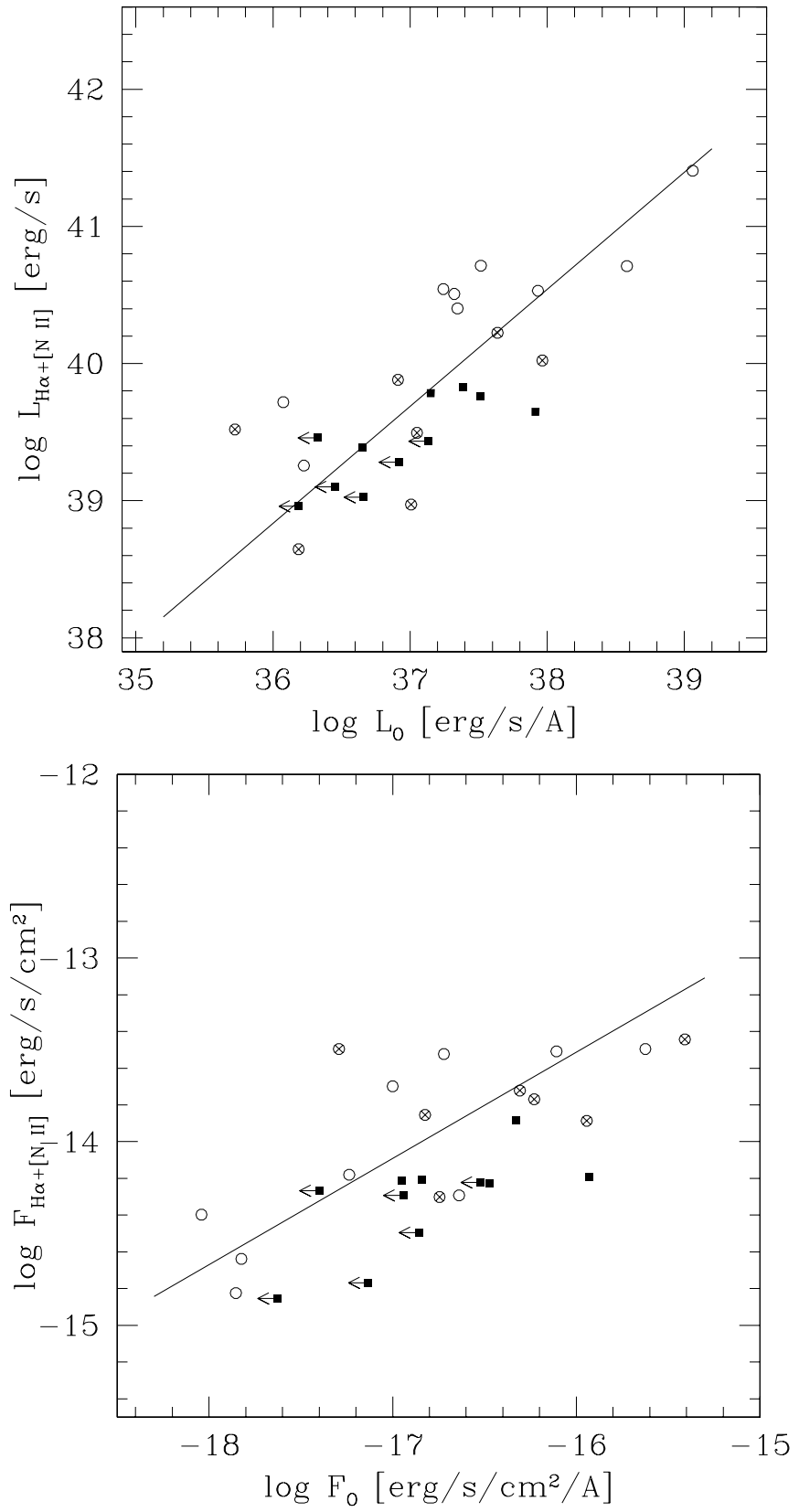

Fig. 2. Relationship between emission line and optical continuum luminosity and flux. Open circles are $3 \mathrm{C}$ sources while filled squares are UGC galaxies. Objects common to the $3 \mathrm{C}$ and UGC samples are marked with a crossed circle. The coefficients of the correlations have been estimated from the $3 \mathrm{C}$ sample alone, but UGC sample clearly follows the same relationship.

\section{The ionization mechanism of the CELR}

The correlation between continuum and line luminosities found in our sample of low luminosity radio-galaxies is reminiscent of the well-known strong connection observed in other classes of AGN (e.g. Adams \& Weedman 1975; Netzer et al. 1992) and similarly suggests a direct link between the optical nuclear sources with the source of ionizing photons responsible for ionization of the CELR. This idea must be tested quantitatively in order to establish if the higher energy photons (UV and soft X-ray) associated to the optical sources provide a 

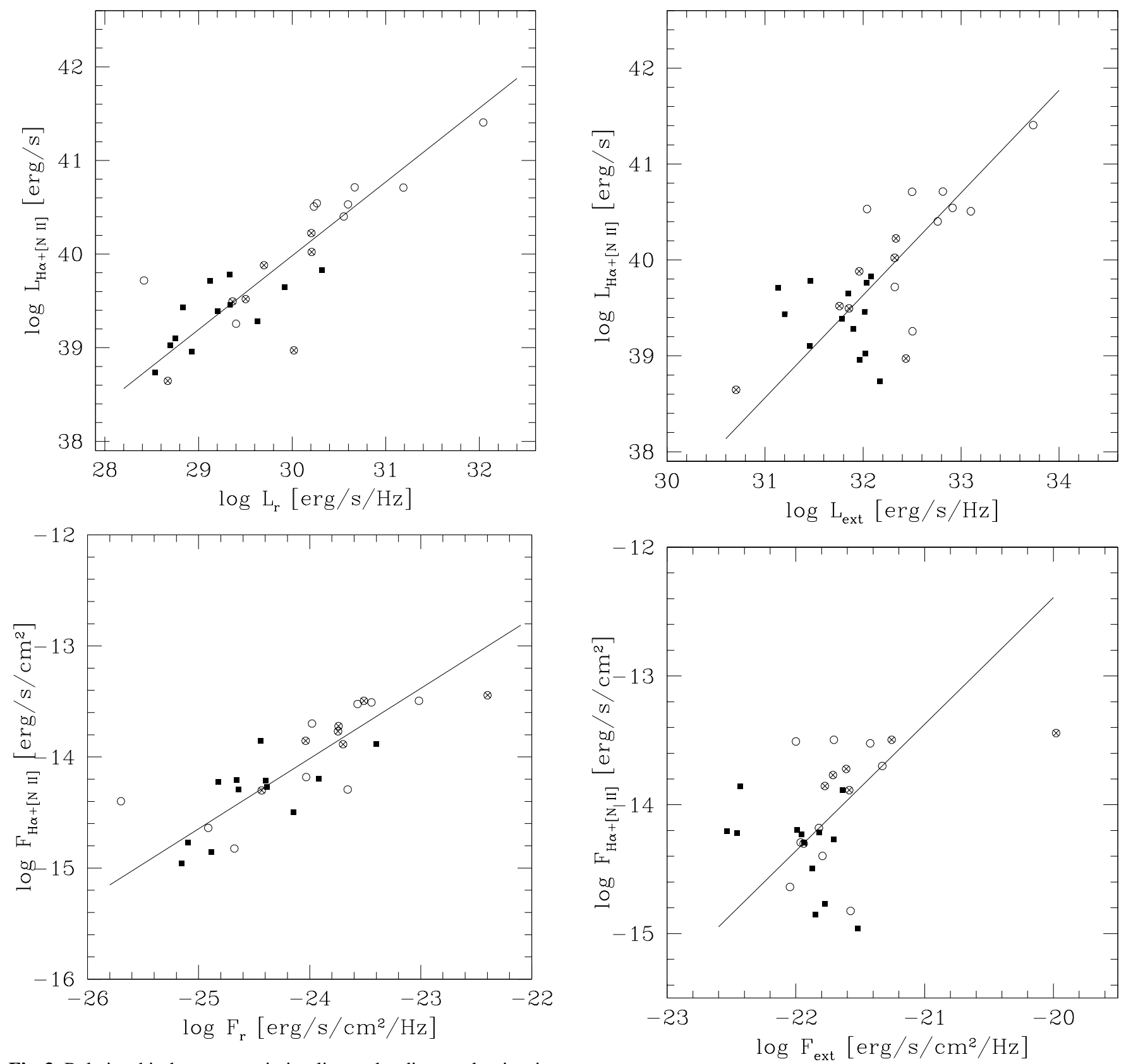

Fig. 3. Relationship between emission line and radio core luminosity and flux. Symbols as in Fig. 2.

sufficient photon flux to ionize the CELR. In the case of LLRG this approach requires particular care due to the paucity of information on their nuclear spectra and the possible effects of anisotropy.

\subsection{The ionizing photons budget for the CELR}

For case B recombination, the flux of Balmer line photons, $Q_{\mathrm{H} \beta}$, is related to the flux of incident ionizing photons, $Q_{\mathrm{ion}}$, as $Q_{\mathrm{H} \beta}=0.12 Q_{\mathrm{ion}} \Omega_{\mathrm{CELR}}$ where $\Omega_{\mathrm{CELR}}$ is the gas-covering factor (Osterbrock 1989). Before applying the photon counting argument, we must convert the $\mathrm{H} \beta$ fluxes to our $\mathrm{H} \alpha+[\mathrm{NII}]$ measurements. We scale the $\mathrm{H} \beta$ to the $\mathrm{H} \alpha$ luminosity adopting the standard 3.1 ratio (e.g. Gaskell \& Ferland 1984). The typical

Fig. 4. Relationship between emission line and extended radio luminosity and flux. Symbols as in Fig. 2.

value of 4 for the [N II] $\lambda \lambda 6584,6548 / \mathrm{H} \alpha$ ratio measured for the UGC sample (Noel-Storr et al. 2003) provides a further factor of 5 correction. In the rest of this section we use $\mathrm{H} \alpha$ to refer to the $\mathrm{H} \alpha+[\mathrm{N} \mathrm{II}]$ complex for the sake of simplicity.

We start with a simple approximation for the nuclear properties assuming i) a power law extrapolation of the optical core luminosity with a constant spectral index $\alpha$, i.e. $L_{v}=$ $C\left(v / v_{\mathrm{opt}}\right)^{-\alpha}$, and ii) isotropy of the nuclear emission. The effects of these assumptions are discussed in detail below. In this case, the flux of ionizing photons is obtained integrating over 


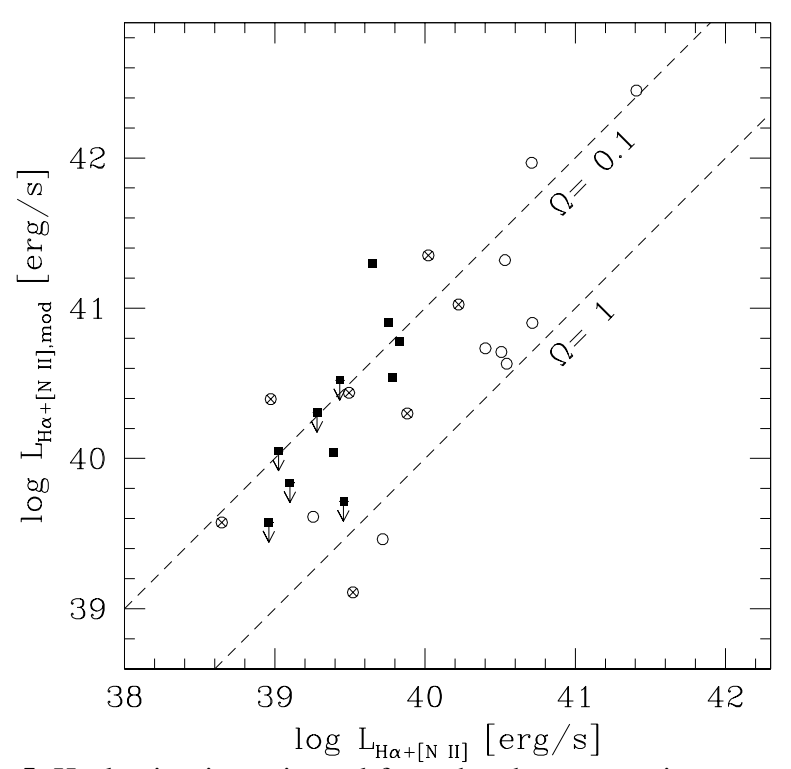

Fig. 5. $\mathrm{H} \alpha$ luminosity estimated from the photon counting argument assuming $\Omega_{\mathrm{CELR}}=1$, compared to the observed luminosity. The ratio between these quantities provides the CELR covering factor, $\Omega_{\mathrm{CELR}}$. Lines of constant $\Omega_{\mathrm{CELR}}$ are shown.

the nuclear spectrum above $v_{0}$, the frequency corresponding to the ionization energy of hydrogen, i.e.

$Q_{\text {ion }}=\int_{v_{0}}^{\infty} \frac{L_{v}}{h v} \mathrm{~d} v=\frac{C}{\alpha h}\left(\frac{v_{0}}{v_{\mathrm{opt}}}\right)^{-\alpha}$ [photons $\mathrm{s}^{-1}$ ].

The spectral indices measured by Donato et al. (2004) for FR I radio-galaxies from the optical to the X-ray are in the range $\alpha_{\mathrm{OX}} \sim 0.8-1.2$. We adopted the median value of 1 for the integration of the nuclear spectrum to estimate the ionizing photon flux. The corresponding model estimates of $\mathrm{H} \alpha$ luminosity, $L_{\mathrm{H} \alpha \mathrm{mod}}$, are compared to the observed ones in Fig. 5. For most objects the covering factors of the CELR $\left(\Omega_{\mathrm{CELR}}\right)$, i.e. the ratio between the observed line luminosity and the one predicted by photon counting, are in the range $\Omega_{\mathrm{CELR}} \sim 0.1-1$ with a median value of 0.28 .

Overall, they compare favorably with the covering factors for the Broad Line Region (BLR) estimated by, e.g., Kinney et al. (1985) for a sample of nearby QSO as they found a range of $0.04-0.47$ with a median of 0.17 . For the Narrow Line Region (NLR) the situation is more complex: as the luminosity of the narrow lines in both Seyfert and QSO is at least 10 times fainter than the corresponding broad components (Boroson \& Green 1992; Osterbrock \& Shuder 1982), a smaller covering factor, a few $10^{-2}$, might be expected. However, this might be a substantial underestimation due to dust embedded in the NLR (as shown by Netzer \& Laor 1993). Nonetheless, the relatively high values of $\Omega_{\text {CELR }}$, although acceptable from a geometrical point of view and subject to the relatively large uncertainties typical of this approach, warrant a more critical assessment of this result.

\subsection{Photons budget and nuclear spectra}

We then estimate how the derived line luminosity $L_{\mathrm{H} \alpha \mathrm{mod}}$, and consequently the covering factors, change when adopting different nuclear spectra. First of all, still in the assumption of a power law spectrum we note that the flux of ionizing photons scales with the spectra index as $\left[\alpha\left(v_{0} / v_{\text {opt }}\right)^{\alpha}\right]^{-1}$. Thus, adopting the extreme observed value of spectral index of 0.8 (1.2), would reduce (increase) the covering factors by a factor of 2 .

To allow for a more realistic input spectrum we also used data for two objects, namely NGC 6251 and Cen A, for which a complete set of nuclear measurements exists over the full energy range, thus allowing detailed analysis of their Spectral Energy Distribution (SED). SED of these two objects have been recently studied by Chiaberge et al. (2001) and Chiaberge et al. (2003) and shown to be well reproduced by a synchrotron self-Compton model. Here we integrate the fit to their SED to estimate the flux of ionizing photons. The advantage of this method is that it allows a better estimate of the contribution of the non thermal nuclear emission also in spectral regions inaccessible to observations, most notably the far UV radiation to which most of the ionizing photons are associated. The effective spectral index (i.e. the power law index $\alpha_{\mathrm{OX}}$ that provides the same number of ionizing photons of the model SED) is 1.6 for Cen A and 1.3 for NGC 6251. These values are slightly higher than the measured $\alpha_{\mathrm{OX}}$ due to the fact that the SED in the UV region is even steeper than predicted by the average $\alpha_{\mathrm{OX}}$. A similar indication of a rather steep spectral shape in the UV regime comes from the HST observations of radiogalaxies discussed by Chiaberge et al. (2002). The lowest value of spectral index measured between 2500 and $7000 \AA$ for FR I radio-galaxies is in fact 1.2 .

\subsection{Photon budget and anisotropy}

The assumption of isotropy for the nuclear emission is clearly another very important point to discuss. In fact, as the radio and (most likely) the optical emission originate from the base of a relativistic jet, dependence on orientation of the nuclear emission is expected. In particular, evidence of a substantial Doppler boosting in the optical core of radio-galaxies was found by CCC99 when comparing the optical and extended radio luminosity of the 3C/FR I: objects seen closer to the jet axis have brighter nuclei for a given radio luminosity. Taking anisotropy into account is therefore essential to a proper ionizing photon budget.

In this context, as already noted by VK02, the relationship between line and continuum luminosity contains two important pieces of information on this issue. Contrary to optical nuclear light, line emission is not affected by beaming and is most likely isotropic. An object seen at increasingly smaller angles from the jet axis increases its nuclear flux at constant line luminosity. This should correspond to a connection between orientation and the location of each object with respect to the $L_{0} / L_{\mathrm{H} \alpha}$ correlation $^{5}$.

5 This trend can also be described as a decrease in the lines' equivalent width at decreasing angles between the jet axis and the line of sight. The very low equivalent widths defining BL Lacs, the most closely aligned objects according to the FR I/BL Lac unified model, would represent the extreme manifestation of this effect. 


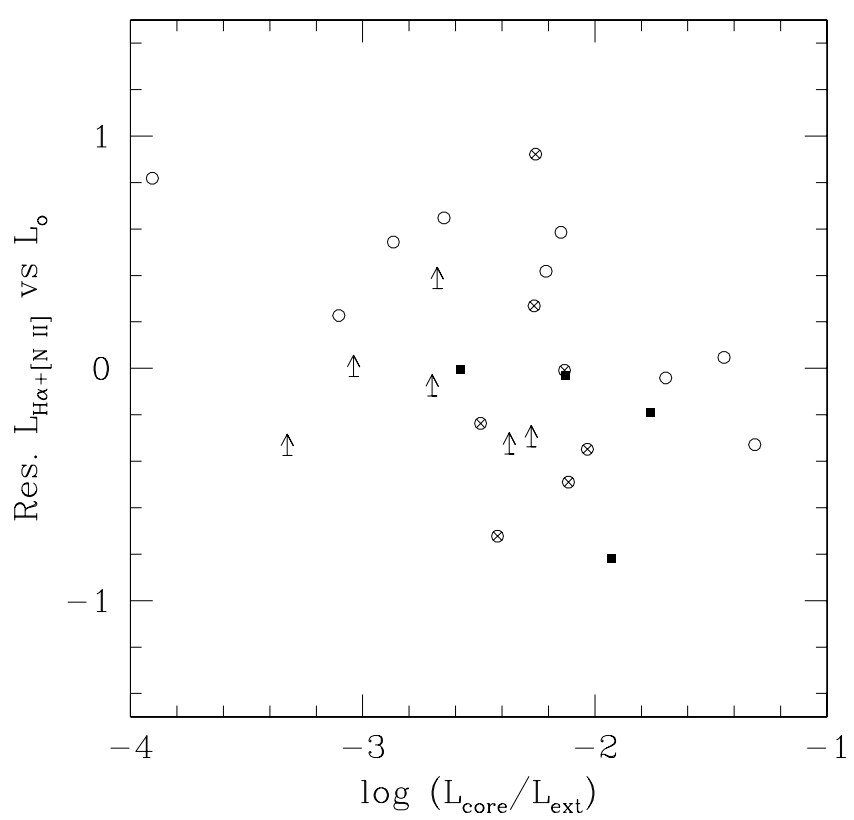

Fig. 6. Residuals of the $L_{\mathrm{H} \alpha}$ vs. $L_{0}$ correlation against the ratio between core and extended radio emission.

To test this idea, we used the radio core dominance, i.e. the ratio between radio core and extended radio luminosity $R=L_{\text {core }} / L_{\text {ext }}$, as indicator of orientation. This ratio has been widely used in the literature to derive the orientation of radio loud AGN despite the relatively large scatter and the known dependence of $R$ on radio power. Nonetheless, Fig. 6 shows the expected trend: objects with larger core dominance value (seen closer to their jet axis) show larger negative residuals from the $L_{0} / L_{\mathrm{H} \alpha}$ correlation; i.e. they have optical sources brighter than average at given emission line luminosity. More quantitatively, with a correlation coefficient of 0.49 , the probability that the 20 detections are taken from a random distribution is $P=0.028$. As continuum and line fluxes are measured essentially at the same wavelengths, this conclusion is independent of dust absorption. This result confirms, first of all, that optical cores are indeed significantly affected by relativistic beaming. Furthermore, this trend shows that at least part of the scatter of the $L_{0} / L_{\mathrm{H} \alpha}$ correlation is due to orientation, then strengthening the connection between line and core luminosity.

A second important result is that the spread of the correlation carries quantitative information concerning the importance of Doppler boosting. Following VK02, this can be compared to the theoretical expectations for the dispersion of a randomly oriented sample of radio-galaxies. Here we have sufficient detection of optical cores to analyze the residuals in this band. Since Doppler amplification depends on the local spectral index $\alpha$, optical data (for which $\alpha \sim 1$ ) set stronger limits on the effects of beaming than do radio measurements (where $\alpha \sim 0)^{6}$. Comparing scatter and residuals of our $L_{0} / L_{\mathrm{H} \alpha}$ correlation with Fig. 6 by VK02 yields a jet bulk Lorentz factor of $\Gamma \sim 2$.

\footnotetext{
${ }^{6}$ The observed flux scales with $\delta^{p+\alpha}$ ( $p=2$ applies to a continuous jet and $p=3$ to a blobby jet), where $\alpha$ is the local spectra index and $\delta=[\Gamma(1-\beta \cos \theta)]^{-1}$ is the Doppler factor, with $\theta$ the angle formed by the jet with the line of sight.
}

Clearly, this value should be considered as an upper limit since other sources of scatter in the correlation are very likely to be present.

Thus, we conclude that relativistic beaming affects the optical nuclei and is then important for a correct photon budget. For example the objects with the largest covering factors can now be understood as those seen at the largest inclinations with the most de-amplified nuclei. However, beaming effects within the small range of $\Gamma$ allowed by the analysis do not substantially affect the photon budget for complete samples. In fact, the median value of $\Omega_{\text {CELR }}$ corresponds to an angle between the line of sight and the jet axis of $60^{\circ}$ (the median value for a randomly distributed population). At this angle the Doppler factor is constrained (for $\Gamma \lesssim 2$ ) to $\delta=[\Gamma(1-0.5 \beta)]^{-1} \lesssim 0.88$ corresponding to a (de)amplification due to Doppler beaming ( $\delta^{p+\alpha}$ with $p=2$ and $\alpha=1$ ) of $\lesssim 0.69$ (and to a decrease in $\Omega_{\mathrm{CELR}}$ by the same factor).

To summarize, extrapolation of the spectra of optical nuclear sources to higher energies corresponds to a sufficient flux of ionizing photons to support the observed line emission, but the re-processing into line photons must be quite efficient, with relatively high CELR covering factors. Anisotropy of the optical nuclei due to Doppler beaming is present and accounts for at least part of the already small scatter between continuum and line luminosities, but the photon budget estimate is rather robust against this effect. The most effective possibility to substantially reduce $\Omega_{\mathrm{CELR}}$ relies on a flattening of the spectral slope toward the UV. But i) this is not expected from the measured optical/X-ray spectral index, ii) it is not observed at least down to the near UV (2500 $⿱$ ) and iii) it is not predicted by the spectral fitting of the two best studied objects.

We conclude that a large CELR covering factor is an unavoidable condition for the viability of this scenario, in which the source of photons ionizing the CELR is directly associated to the optical nuclear sources.

\section{A physical picture of the compact ELR in radio-galaxies}

HST images and spectra of the CELR of LLRG also provide us with important information concerning their physical structure. In the following we focus on 3C 274 (M 87), the closest and best studied object of the sample.

First of all, the size of the compact emitting region in M 87 is constrained by the HST images to be smaller than $R_{\text {CELR }}<$ $0{ }^{\prime} 05 \sim 3 \mathrm{pc}$, as this is unresolved at 0 . $^{\prime} 1$ resolution. The electrons density in its central emission line region has been derived by Macchetto et al. (1997) from HST/FOS spectra measuring the ratio of the sulfur lines [S II] $\lambda 6731 /[$ S II] $\lambda 6717$. As we move toward the nucleus this ratio decreases reaching $0.6 \pm 0.1$ at the central aperture, still far from the ratio that corresponds to the high density limit (0.42). This is indicative of an increase in density from a few hundreds to about $\sim 4300 \mathrm{~cm}^{-3}$ (with a factor of 2 accuracy) at the nucleus. 
As discussed in Osterbrock (1989), the mass of ionized gas can be estimated from the observed Balmer line luminosity ${ }^{7}$. For $L_{\mathrm{H} \alpha+[\mathrm{NII}]}=2 \times 10^{39} \mathrm{erg} / \mathrm{s}$ and the same scaling used above to convert to $L_{\mathrm{H} \beta}$, we obtain a mass of $M=120\left(10^{4} / n_{\mathrm{e}}\right) M_{\odot}$. Combining these measurements with the limit to the CELR volume, we derive the filling factor for the ELR gas of $\epsilon \sim 0.03$ for the above measured density. This value is actually a lower limit (as the measured ELR radius is an upper limit), and it also indicates that the size of the CELR cannot be substantially smaller than $\sim 1 \mathrm{pc}$, as the filling factor would exceed unity.

Limits on the CELR's size for the other more distant sources are less stringent, but nonetheless they are constrained to be smaller than $\sim 20 \mathrm{pc}$ for $\sim 80 \%$ of the sample. Scaling the mass found for M 87 to the range of LLRG line luminosity yields a typical CELR mass of $10-10^{3} M_{\odot}$.

Another important piece of information concerns the dynamics of the nuclear gas. Macchetto et al. (1997) showed that the gas rotation curve can be accurately reproduced as Keplerian motions of gas arranged in a thin disk around the central super-massive black hole. This result is common to several other objects of our 3C sample, e.g. 3C 272.1 (Bower et al. 1998), NGC 6251 (Ferrarese \& Ford 1999), 3C 270 (Ferrarese et al. 1996), by two UGC sources, UGC 11718 (i.e. NGC 7052, van der Marel \& van den Bosch 1998) and UGC 7455 (Verdoes Kleijn et al. 2002b), as well as in another LLRG, Centaurus A (Marconi et al. 2001).

Although clearly we do not have information on the dynamics (or on the morphology) of the CELR, the Keplerian motion can be followed from $\sim 100 \mathrm{pc}$ down to the HST resolution limit, $5 \mathrm{pc}$ in the case of M 87. On the other hand, as discussed above, the radius of its CELR cannot be substantially smaller than $\sim 1 \mathrm{pc}$. If the disk structure is also preserved on the smaller scales, the estimated covering factor of CELR requires a substantial vertical thickness of the gaseous disk: for example, a value of $\Omega_{\text {CELR }} \sim 0.3$ corresponds to an angular thickness of $\theta \sim 15^{\circ}$. In this sense, the central emission line regions would probably be better described as ionized tori.

This raises the issue of the vertical support for the CELR. While thermal motions are negligible in this context, detailed model fitting of circumnuclear disks around black holes show that the measured velocity dispersions are generally in excess of what it is predicted from just spatial smearing (e.g. Verdoes Kleijn et al. 2000; van der Marel \& van den Bosch 1998; Barth et al. 2001). This requires the presence of an internal velocity component in addition to thermal and rotational motions, possibly due to turbulence, reaching in the innermost regions values of several hundreds of $\mathrm{km} \mathrm{s}^{-1}$, comparable to the rotational velocity at these radii. While this line of argument does not answer the question of the physical origin of the

7 The mass and filling factor of ionized gas are given by

$M=\frac{m_{\mathrm{p}}+0.1 m_{\mathrm{He}}}{n_{\mathrm{e}} \alpha_{\mathrm{H} \beta}^{\mathrm{eff}} h \nu_{\mathrm{H} \beta}} L_{\mathrm{H} \beta} ; \quad \epsilon=\frac{M}{\left(m_{\mathrm{p}}+0.1 m_{\mathrm{He}}\right) V}$

where $V$ is the volume of the emitting region. We adopted a temperature of $10^{4} \mathrm{~K}$, i.e. a recombination coefficient $\alpha_{\mathrm{H} \beta}^{\mathrm{eff}}=3.03 \times$ $10^{-14} \mathrm{~cm}^{3} \mathrm{~s}^{-1}$. vertical support, it does show that this can be provided by turbulent velocity.

\section{Line emission vs. radio-luminosity}

Line and radio luminosity (both core and total radio power) are known to correlate for the powerful FR II radio-galaxies (e.g. Rawlings \& Saunders 1991; Zirbel \& Baum 1995; Willott et al. 1999). Results presented in Sect. 3 show a correlation between $L_{\text {core }}$ and $L_{\mathrm{H} \alpha}+$ [N II], which contrasts with previous ground-based determination of these relationships by Zirbel \& Baum (1995), as they found a much flatter slope $(0.30 \pm 0.12)$ with a larger dispersion, 0.79 dex. The most likely explanation for this discrepancy is contamination of the emission line luminosity by the host galaxy, particularly for the faintest objects. In fact, FR I appears to be smoothly connected to nonactive galaxies, as far as their line and radio luminosity is concerned. Non-active galaxies also show a correlation between NLR and host galaxy's luminosity. Only in the brightest FR I does the AGN component make a substantial contribution to the total line emission and induce the somewhat loose trend they observed. By solely concentrating on a well-defined sample of relatively bright FR I and isolating the central emission line component, a clearer view of the connection between these quantities emerges.

The interpretation of the radio-core/optical correlation observed in our sample of LLRG FR I appears to be rather straightforward in the scenario presented in the previous sections. The line luminosity correlates with the optical continuum as this is directly associated with the source of ionizing photons; the optical continuum is most likely of synchrotron origin and it is produced by the same mechanism as the radio core.

\section{Discussion}

Broad-band HST images of LLRG revealed the presence of a correlation between fluxes and luminosities in the nuclear optical sources with the corresponding radio cores, thus indicating a dominant non-thermal (jet) origin for their optical emission. Narrow-band images (and spectra) of LLRG also show a strong correlation between fluxes and luminosities of the optical sources with those of their compact emission-line regions. A photon budget argument supports the idea that these nonthermal nuclei provide a sufficient flux of ionizing photons, i.e. that we are seeing a jet-ionized CELR.

The origin of the lack of prominent broad lines in LLRG must also be revised in the light of the present results. In fact high density clouds in the presence of the ionizing photon fields required to illuminate the compact ELR (regardless of their origin) should correspond to a significant broad line flux. With a simple scaling argument, adopting a BLR density of $n_{\mathrm{e}}=10^{9} \mathrm{~cm}^{-3}$, the BLR luminosity would exceed the CELR luminosity as soon as $M_{\mathrm{BLR}} / M_{\mathrm{CELR}} \gtrsim n_{\mathrm{e}, \mathrm{CELR}} / n_{\mathrm{e}, \mathrm{BLR}}$, assuming that both structures have similar covering factors. This sets a limit of $M_{\mathrm{BLR}}<10^{-2} M_{\odot}$, several orders of magnitude below the BLR mass estimated for luminous AGN (see Baldwin et al. 2003 for a detailed discussion of BLR masses). 
Table 3. Properties of the $3 \mathrm{C}$ sample.

\begin{tabular}{lccccrrrr}
\hline \hline & UGC name & VK02 & NGC name & $z$ & $F_{\mathrm{r}}$ & $F_{\text {rext }}$ & $F_{\text {opt }}$ & $F_{\mathrm{H} \alpha+[\mathrm{NII}]}$ \\
\hline 3C 029 & 595 & & & 0.0448 & 93 & 15.1 & $5.8 \mathrm{e}-18$ & $6.6 \mathrm{e}-15$ \\
3C 031 & 689 & yes & 383 & 0.0169 & 92 & 16.8 & $1.5 \mathrm{e}-17$ & $1.4 \mathrm{e}-14$ \\
3C 066B & 1841 & yes & & 0.0215 & 182 & 24.6 & $4.9 \mathrm{e}-17$ & $1.9 \mathrm{e}-14$ \\
3C 078 & 2555 & & 1218 & 0.0290 & 964 & 19.8 & $2.4 \mathrm{e}-16$ & $3.2 \mathrm{e}-14$ \\
3C 083.1 & 2651 & & 1265 & 0.0251 & 21 & 26.6 & $1.4 \mathrm{e}-18$ & $1.5 \mathrm{e}-15$ \\
3C 264 & 6723 & yes & 3862 & 0.0206 & 200 & 26.0 & $1.1 \mathrm{e}-16$ & $1.3 \mathrm{e}-14$ \\
3C 270 & 7360 & yes & 4261 & 0.0074 & 308 & 55.5 & $5.1 \mathrm{e}-18$ & $3.2 \mathrm{e}-14$ \\
3C 272.1 & 7494 & yes & 4374 & 0.0037 & 180 & 19.4 & $5.9 \mathrm{e}-17$ & $1.7 \mathrm{e}-14$ \\
3C 274 & 7654 & yes & 4486 & 0.0037 & 4000 & 1050.0 & $3.9 \mathrm{e}-16$ & $3.6 \mathrm{e}-14$ \\
3C 277.3 & & & & 0.0857 & 12 & 9.0 & $1.5 \mathrm{e}-18$ & $2.3 \mathrm{e}-15$ \\
3C 338 & 10409 & & 6166 & 0.0303 & 105 & 46.9 & $1.0 \mathrm{e}-17$ & $2.0 \mathrm{e}-14$ \\
3C 346 & & & & 0.1620 & 220 & 10.9 & $2.3 \mathrm{e}-17$ & $5.1 \mathrm{e}-15$ \\
3C 442 & 11958 & & & 0.0262 & 2 & 16.1 & $9.1 \mathrm{e}-19$ & $4.0 \mathrm{e}-15$ \\
3C 449 & 12064 & yes & & 0.0181 & 37 & 11.5 & $1.8 \mathrm{e}-17$ & $5.0 \mathrm{e}-15$ \\
3C 465 & 12716 & & 7720 & 0.0301 & 270 & 37.8 & $1.9 \mathrm{e}-17$ & $3.0 \mathrm{e}-14$ \\
NGC 6251 & 10501 & & 6251 & 0.0240 & 360 & 10.0 & $7.8 \mathrm{e}-17$ & $3.1 \mathrm{e}-14$ \\
\hline
\end{tabular}

$F_{\mathrm{r}}$ radio core flux at $5 \mathrm{GHz}$ in mJy, $F_{\text {rext }}$ total radio flux at $178 \mathrm{MHz}$ in Jy, $F_{\text {opt }}$ optical core flux at $7020 \AA$ in erg/s/cm ${ }^{2} / \AA, F_{\mathrm{H} \alpha+[\mathrm{NII}]}$ flux of the compact emission line region in $\mathrm{erg} / \mathrm{s} / \mathrm{cm}^{2}$.

Table 4. Properties of the UGC sample.

\begin{tabular}{lccrrrr}
\hline \hline & NGC name & $z$ & $F_{\mathrm{r}}$ & $F_{\text {rext }}$ & $F_{\text {opt }}$ & $F_{\mathrm{H} \alpha+[\mathrm{NII}]}$ \\
\hline UGC 00408 & 193 & 0.0145 & 40 & 3.4 & $1.1 \mathrm{e}-17$ & $6.1 \mathrm{e}-15$ \\
UGC 00597 & 315 & 0.0165 & 396 & 5.2 & $4.7 \mathrm{e}-17$ & $1.3 \mathrm{e}-14$ \\
UGC 01004 & 541 & 0.0181 & 8 & 3.8 & $<7.4 \mathrm{e}-18$ & $1.7 \mathrm{e}-15$ \\
UGC 01413 & 741 & 0.0185 & 13 & 3.2 & $<2.4 \mathrm{e}-18$ & $1.4 \mathrm{e}-15$ \\
UGC 03695 & 2329 & 0.0191 & 117 & 2.3 & $1.2 \mathrm{e}-16$ & $6.4 \mathrm{e}-15$ \\
UGC 05073 & 2892 & 0.0227 & 22 & 0.7 & $1.4 \mathrm{e}-17$ & $6.2 \mathrm{e}-15$ \\
UGC 06635 & 3801 & 0.0109 & - & 3.4 & - & - \\
UGC 07115 & & 0.0226 & - & 2.5 & $3.4 \mathrm{e}-17$ & $5.9 \mathrm{e}-15$ \\
UGC 07455 & 4335 & 0.0154 & 15 & 7.9 & $<3.0 \mathrm{e}-17$ & $6.0 \mathrm{e}-15$ \\
UGC 08419 & 5127 & 0.0161 & 7 & 0.7 & - & $1.1 \mathrm{e}-15$ \\
UGC 08433 & 5141 & 0.0177 & 71 & 3.0 & $<1.4 \mathrm{e}-17$ & $3.2 \mathrm{e}-15$ \\
UGC 09058 & 5490 & 0.0167 & 41 & 4.4 & $<4.0 \mathrm{e}-18$ & $5.4 \mathrm{e}-15$ \\
UGC 11718 & 7052 & 0.0139 & 36 & 0.8 & - & $1.4 \mathrm{e}-14$ \\
UGC 12531 & 7626 & 0.0114 & 23 & 2.6 & $<1.1 \mathrm{e}-17$ & $5.1 \mathrm{e}-15$ \\
\hline
\end{tabular}

$F_{\mathrm{r}}$ radio core flux at $1.4 \mathrm{GHz}$ in mJy, $F_{\text {rext }}$ total radio flux at $408 \mathrm{MHz}$ in Jy, $F_{\text {opt }}$ optical core flux at $8140 \AA$ in $\operatorname{erg} / \mathrm{s} / \mathrm{cm}^{2} / \AA ̊, F_{\mathrm{H} \alpha+[\mathrm{NII}]}$ flux of the compact emission line region in $\mathrm{erg} / \mathrm{s} / \mathrm{cm}^{2}$.

We conclude that in LLRG the dense clouds commonly associated to the BLR are virtually not present. A possible explanation for this finding has been proposed by Laor (2003): given the relationship between BLR radius, $R_{\mathrm{BLR}}$, and nuclear luminosity, $R_{\mathrm{BLR}}$ would be smaller than the minimum radius for clouds surviving tidal disruption below a bolometric luminosity of $L \sim 10^{41.8}\left(M_{\mathrm{BH}} / 10^{8} M_{\odot}\right)^{2}$, a condition met by our LLRG.

The low masses of both ELR and BLR represent further evidence of the paucity of gas in the innermost regions of LLRG, to be added to the low accretion rate (derived from the low nuclear luminosity), to the failure of finding maser emission (Henkel et al. 1998), and to the general lack of obscuring molecular tori.

The available information indicates that the CELR must have substantial thickness to provide the necessary covering factors. If the disky structure of the emission-line region observed at larger radii is preserved down to the pc-scale, the CELR would be better described as ionized tori. This raises the question of how these structures would compare with the molecular tori of other classes of AGN. The low fraction of 
possibly optically obscured LLRG ( $15 \%$, CCC99) suggests that little dust is associated to the CELR. More quantitatively, HST ultraviolet observations (Chiaberge et al. 2002) have provided evidence for a relationship between UV absorption and orientation as expected from a flattened absorber. For sources seen close to the equatorial plane its optical depth corresponds to $A_{\mathrm{V}} \sim 3$.

The arguments presented in Sect. 5 enable us to also estimate their ionized gas column density that can be compared with results from X-ray observations. The average density of ionized gas derived from a spherical distribution is $\left\langle n_{\mathrm{e}}\right\rangle \sim 10^{2} \mathrm{~cm}^{-3}$ corresponding to a column density of $N_{\mathrm{H}} \sim$ $\left\langle n_{\mathrm{e}}\right\rangle r_{\mathrm{CELR}} \sim 10^{21} \mathrm{~cm}^{-2}$. This represents an average value for a randomly oriented class of objects, but if the absorbing material is partially flattened, we should be seeing a fraction of LLRG along lines of sight that do not intercept the torus, while for the remaining sources a larger $N_{\mathrm{H}}$ should be measured. This scenario is indeed supported by recent X-ray observations of LLRG providing examples of moderate photoelectric absorption (e.g. Sambruna et al. 2000, 2003) with typical column density of a few $10^{22} \mathrm{~cm}^{-2}$ contrasted by the majority of objects in which no absorption in excess to the Galactic component is found (e.g. Hardcastle et al. 2001, 2002). In this sense the most significant difference between the putative tori in LLRG and those of Seyfert would be the much lower optical depth: in fact $\sim 75 \%$ of Seyfert have $N_{\mathrm{H}}>10^{23} \mathrm{~cm}^{-2}$ and about half are Compton-thick with $N_{\mathrm{H}}>10^{24} \mathrm{~cm}^{-2}$ (Risaliti et al. 1999). The resulting effects of the circumnuclear gas in LLRG would be radically different from those of the standard molecular tori. Not only does it allow a direct view of the nucleus, but it provides a preferred environment for the production of line emission.

It would also be interesting at this point to establish if a CELR is present in other AGN as well, as this would bring important information on their nuclear structure. Most likely it can be directly viewed only in type I sources. Indeed, the excess of high-ionization coronal lines, such as [Fe VII], in Sy 1 with respect to Sy 2 (Murayama \& Taniguchi 1998) has been interpreted as an indication that they are produced (for a large fraction of the total flux) at the inner wall of the dusty torus. This is analogous to the situation envisaged to explain the properties of LLRG, albeit in a different regime of photons and gas density.

Alternative interpretations, such a standard disk ionization, clearly can not be excluded at this stage, despite the internal consistency of the jet-ionized CELR scenario. However, in this latter case the strong correlation between the optical core, radio core and CELR luminosity, would imply a close link between the thermal radiation from the disk and non-thermal radiation from the jet. While a general link between these two processes might be expected (e.g. Falcke et al. 1995), such a close connection appears contrived, but cannot be discarded at face value.

A further hint as to the nature of the ionization source in LLRG comes from their optical spectra, which are invariably of the LINER type. It has been suggested that a LINER spectrum can arise from a low ionization parameter $(U=$ $Q_{\text {ion }} /\left(4 \pi r^{2} n_{\mathrm{e}} c\right) \sim 10^{-3}-10^{-4}$ e.g. Ferland \& Netzer 1983).
However, estimates for M 87 based on the values presented in Sect. 5 sets it at a substantially higher value as we find $U \sim 0.008 \Omega_{\mathrm{CELR}}{ }^{-1}$ for a CELR radius of $3 \mathrm{pc}$. As an alternative, Nagao et al. (2002) recently showed that harder spectra than those emitted by standard optically thick accretion disks produce larger partially ionized regions, increasing the relative weight of low ionization lines. Clearly, a detailed spectroscopic study and modeling of CELRs is needed to investigate this issue.

\section{Summary and conclusions}

With the aim of studying the origin and properties of the emission line region of Low Luminosity Radio Galaxies, we collected HST spectra and narrow-band images for a total of 30 sources. HST images show that a CELR is present at the center of all objects studied, with just one exception. Focusing on this central compact component, we minimize the contamination from line emission not directly related to the AGN, which can be significant in these low luminosity objects. A clearer view of the LLRG properties emerges by isolating the central emission line component.

The line luminosities show a strong connection to other properties of these objects, most importantly it correlates over almost four orders of magnitude with the optical nuclear luminosity. This trend is reminiscent of what is observed in other classes of more powerful AGN and similarly suggests a direct link between the optical nuclear sources and the source of the photons ionizing the NLR. Extrapolation of the spectra of optical nuclear sources to higher energies corresponds to a sufficient flux of ionizing photons, but requires relatively high covering factors with a median value of 0.28 . We tested the effects both of different choices for the nuclear spectra and of anisotropy of the optical nuclei. Beaming effects are present, but within the small range allowed (the bulk jet Lorentz factor is constrained to be $\Gamma \lessgtr 2$ ) they do not substantially affect the photon budget.

HST images and spectra of the CELR of LLRG also provide us with information concerning their physical structure. Using observations of 3C 274 (M 87) we derive the following fiducial values: a limit to the NLR size of $\sim 3 \mathrm{pc}$, a density of $\sim 4000 \mathrm{~cm}^{-3}$, a mass $10^{2} M_{\odot}$, and a filling factor larger than $\epsilon \sim 0.03$. Furthermore, the dynamics of the nuclear gas in LLRG is accurately reproduced as Keplerian motions around the central super-massive black hole in many objects of our sample down to a scale of a few parsec. If the disky structure of the emission line region observed at larger radii is preserved down to the pc-scale, the covering factor derived from the photon-budget can be accounted for if the CELR has an angular thickness of at least $\theta \sim 15^{\circ}$. In this sense the CELR would be better described as ionized tori. Indeed, detailed modeling of circumnuclear disks around black holes often requires the presence of a large component of turbulent velocity providing the disk's vertical support.

Our conclusions about the physical structure of the CELR do not depend on the origin of the ionizing photons. In particular we find that its mass is as small as $10-10^{3} M_{\odot}$ while any BLR mass must be smaller than $M_{\mathrm{BLR}}<10^{-2} M_{\odot}$; 
i.e. the dense clouds commonly associated to the BLR are virtually not present. When considered together with the low accretion rate and to the general lack of obscuring molecular tori, these results represent further evidence that the different behaviour of LLRG from the overall AGN population can be ascribed to the paucity of gas in their innermost regions.

The internal consistency of the jet-ionized CELR scenario clearly cannot be used to rule out alternative interpretations, although the strong correlation of optical core vs. line luminosity requires a similarly strong connection between any other sources of ionizing photons with the jet emission, which appears to be quite contrived.

CELR luminosity also shows a quasi-linear correlation with the radio core that contrasts with a much flatter slope and larger dispersion than in previous determination of this relationship. The most likely explanation for these discrepancies is the contamination of the emission line luminosity by a nonAGN extended component. Concentrating on a well-defined sample of relatively bright FR I and isolating the central emission line, a clearer view of the connection between these quantities emerges. Interpretation of the radio-core/line correlations observed in FR I is rather straightforward in the proposed scenario of a jet-illuminated NLR as the result of the chain of correlations linking line emission, optical cores, and radio cores.

\section{References}

Adams, T. F., \& Weedman, D. W. 1975, ApJ, 199, 19

Auriemma, C., Perola, G. C., Ekers, R. D., et al. 1977, A\&A, 57, 41

Baldwin, J. A., Ferland, G. J., Korista, K. T., Hamann, F., \& Dietrich, M. 2003, ApJ, 582, 590

Barth, A. J., Sarzi, M., Rix, H., et al. 2001, ApJ, 555, 685

Boroson, T. A., \& Green, R. F. 1992, ApJS, 80, 109

Bower, G. A., Green, R. F., Danks, A., et al. 1998, ApJ, 492, L111

Chiaberge, M., Capetti, A., \& Celotti, A. 1999, A\&A, 349, 77

Chiaberge, M., Capetti, A., \& Celotti, A. 2001, MNRAS, 324, L33

Chiaberge, M., Gilli, R., Capetti, A., \& Macchetto, F. D. 2003, ApJ, 597, 166

Chiaberge, M., Macchetto, F. D., Sparks, W. B., et al. 2002, ApJ, 571, 247

Donato, D., Sambruna, R. M., \& Gliozzi, M. 2004, ApJ, 617, 915

Dopita, M. A., Koratkar, A. P., Allen, M. G., et al. 1997, ApJ, 490, 202

Falcke, H., Malkan, M. A., \& Biermann, P. L. 1995, A\&A, 298, 375

Ferland, G. J., \& Netzer, H. 1983, ApJ, 264, 105

Ferrarese, L., \& Ford, H. C. 1999, ApJ, 515, 583

Ferrarese, L., Ford, H. C., \& Jaffe, W. 1996, ApJ, 470, 444
Gaskell, C. M., \& Ferland, G. J. 1984, PASP, 96, 393

Hardcastle, M. J., Birkinshaw, M., \& Worrall, D. M. 2001, MNRAS, 326,1499

Hardcastle, M. J., Worrall, D. M., Birkinshaw, M., Laing, R. A., \& Bridle, A. H. 2002, MNRAS, 334, 182

Henkel, C., Wang, Y. P., Falcke, H., Wilson, A. S., \& Braatz, J. A. 1998, A\&A, 335, 463

Ho, L. C., Filippenko, A. V., \& Sargent, W. L. W. 1997, ApJS, 112, 315

Kinney, A. L., Huggins, P. J., Bregman, J. N., \& Glassgold, A. E. 1985, ApJ, 291, 128

Laing, R. A., Riley, J. M., \& Longair, M. S. 1983, MNRAS, 204, 151

Laor, A. 2003, ApJ, 590, 86

Macchetto, F., Marconi, A., Axon, D. J., et al. 1997, ApJ, 489, 579

Marconi, A., Capetti, A., Axon, D. J., et al. 2001, ApJ, 549, 915

Miller, C. J., Nichol, R. C., Gómez, P. L., Hopkins, A. M., \& Bernardi, M. 2003, ApJ, 597, 142

Murayama, T., \& Taniguchi, Y. 1998, ApJ, 497, L9

Nagao, T., Murayama, T., Shioya, Y., \& Taniguchi, Y. 2002, ApJ, 567, 73

Netzer, H., \& Laor, A. 1993, ApJ, 404, L51

Netzer, H., Laor, A., \& Gondhalekar, P. M. 1992, MNRAS, 254, 15

Noel-Storr, J., Baum, S. A., Verdoes Kleijn, G., et al. 2003, ApJS, 148, 419

O’Dea, C. P., Koekemoer, A. M., Baum, S. A., et al. 2001, AJ, 121, 1915

Osterbrock, D. E. 1989, Astrophysics of Gaseous Nebulae and Active Galactic Nuclei (Mill Valley: Univ. Sci. Books)

Osterbrock, D. E., \& Shuder, J. M. 1982, ApJS, 49, 149

Rawlings, S., \& Saunders, R. 1991, Nature, 349, 138

Risaliti, G., Maiolino, R., \& Salvati, M. 1999, ApJ, 522, 157

Sambruna, R. M., Chartas, G., Eracleous, M., Mushotzky, R. F., \& Nousek, J. A. 2000, ApJ, 532, L91

Sambruna, R. M., Gliozzi, M., Eracleous, M., Brandt, W. N., \& Mushotzky, R. 2003, ApJ, 586, L37

van der Marel, R. P., \& van den Bosch, F. C. 1998, AJ, 116, 2220

Verdoes Kleijn, G. A., Baum, S. A., de Zeeuw, P. T., \& O'Dea, C. P. 1999, AJ, 118, 2592

Verdoes Kleijn, G. A., van der Marel, R. P., Carollo, C. M., \& de Zeeuw, P. T. 2000, AJ, 120, 1221

Verdoes Kleijn, G. A., Baum, S. A., de Zeeuw, P. T., \& O'Dea, C. P. 2002a, AJ, 123, 1334

Verdoes Kleijn, G. A., van der Marel, R. P., de Zeeuw, P. T., Noel-Storr, J., \& Baum, S. A. 2002b, AJ, 124, 2524

Willott, C. J., Rawlings, S., Blundell, K. M., \& Lacy, M. 1999, MNRAS, 309, 1017

Xu, C., Baum, S. A., O’Dea, C. P., Wrobel, J. M., \& Condon, J. J. 2000, AJ, 120, 2950

Zirbel, E. L., \& Baum, S. A. 1995, ApJ, 448, 521 